\title{
When Will The Biology of Aging Become Useful? Future Landmarks in Biomedical Gerontology
}

\author{
Richard A. Miller, MD, $P h D$
}

\begin{abstract}
Where should we look to find the causes of and cure for aging? This essay considers a number of scientific discoveries, not yet made, that might dramatically increase the proportion of biogerontologists doing useful work. I will consider, in turn, problems and prospects in the areas of comparative biology, mammalian and invertebrate genetics, biomarker research, caloric restriction, and clonal senescence and then conclude with a discussion of potential links between aging and late life disease. J Am Geriatr Soc 45:1258-1267, 1997.
\end{abstract}

B iogerontologists - members of the small cadre of researchers obsessed with the basic biology of aging and its putative links to disease - cannot yet look you straight in the eye and state honestly that they are doing useful research, research that is bound to reveal the secrets of aging and thereby lead to improvements in human well-being. Aging, despite centuries of descriptive work and decades of progressively more thoughtful hypothesis-driven studies, and despite the fascination that the topic holds for lay and professional public alike, has not yet taken the step up from mystery to problem. Infection before the discovery of microbes, optics before the discovery of the photon, genetics before the double helix, astronomy before the law of gravitation: all were mysteries in the same sense that biogerontology is today, i.e., domains of study in which thoughtful, well-read, industrious investigators could not be certain that their particular tunnel had a light at the other end. At a time when many biomedical journals are devoting increasing attention to the aged and to aging, to gerontology and to geriatric medicine, it seems appropriate to compile a list of imaginary future landmarks, a set of scientific results that have not yet been discovered but whose discovery might break open the aging problem and convert it into a topic suitable for lucid discourse, a mappable terrain for exploration.

Before discussing cases, it will be helpful to venture a definition of a tendentious term, not to settle controversies ex cathedra but to provide a common vocabulary throughout this essay. I will use the term aging to refer to the process that

From the Geriatrics Center, Department of Pathology, and Institute of Gerontology, University of Michigan, and Ann Arbor DVA Medical Center, Ann Arbor, Michigan.

Work cited in this essay was supported by Grants AG11678 and AG11067. Address correspondence to Richard A. Miller, MD, PhD, Medical Sciences Research, Bldg III Rm. 6240, The Geriatrics Center, University of Michigan, 1150 W. Medical Center Dr., Ann Arbor, MI 48109-0642. gradually turns healthy adults - teen-age humans or 8 week old mice - into frail older individuals with a diminished capacity for surviving threats, including infectious agents, the stress of surgery or cold weather, or physical dangers such as onrushing buses or owls. This definition excludes, arbitrarily, perhaps, though I think in a helpful way, such phenomena as (1) the aging of cheese, wine, and beef, (2) the senescence process that leads to leaf abscission, (3) the aging of erythrocytes that leads to their removal from circulation, (4) the processes that convert embryos into babies and babies into teen-agers, (5) apoptosis or programmed cell death, (6) what happens to salmon after upstream mating, and (7) the process that converts early passage diploid cells into late passage cells incapable of further cell division. All of the phenomena on this exclusion list are highly interesting and deserve intensive study, and it may that the exploration of one or more of these processes will lead to improved understanding of biological aging per se. Nothing is gained, however, from the confusion that can result from using the term aging indiscriminately to refer to some ill-defined subset of the phenomena listed above. The ideas that embryogenic development sets seeds that later bloom into senescent changes, or that clonal senescence underlies some or all late-life illnesses, or that postreproductive decline in semelparous fish might provide insights into aging as it affects most mammals - ideas of this kind require careful specification and testing, an analytical process that is impeded by the reckless assumption that they are "merely different varieties of aging."

A second, related semantic obstacle comes from the intimate connection between aging and disease, often encapsulated in the assertion (from a physician colleague) that "aging is just disease" or (from a veterinary pathologist) that "aging is just the sum of lesions." These perspectives confuse process with result. While analysis of the pathogenesis of specific illnesses and specific lesions is undoubtedly a worthy occupation, the key challenge for biogerontology seems to me to discover the basis for intraspecies synchrony (and interspecies homology) among the many diseases and lesions, and the subclinical declines and predictable changes in tissue architecture that accumulate in old age. Atherosclerotic compromise of key circulatory pathways, bothersome loss of muscle strength and joint flexibility, slowed reflexes and slowed immune responses, declines in one or more special senses, diminished short-term memory and processing speed, altered glucose tolerance and fat metabolism, preneoplastic changes or frank neoplasia: although no 80 year-old person (or 3 year old mouse) will exhibit all such changes, it is very rare to find an aged individual who exhibits none of these signs, and 
equally rare to find a 16 year-old person bothered by any of them. The special challenge for biological aging research is to figure out why it is so easy to distinguish teen-agers from their grandparents, to discover the hidden links among these disparate disadvantages, and to discover why humans can avoid for decades the pathophysiological decline that afflicts 2year-old mice and 10-year-old dogs.

It is now unfashionable to refer to aging as a single process and much more acceptable to consider aging as a group of processes that work in parallel. ${ }^{1}$ The idea of multiple processes has much to recommend it because it is indeed difficult to suggest a single influence that might lead simultaneously to such diverse outcomes as changes in cross-linking of extracellular proteins and altered gene expression in many cell types, to hyperproliferation of cells we could do without, to poor proliferation of cells we need, and to diminished responsiveness in cells that do not proliferate at all. And yet the coordinated retardation of this whole set of processes has indeed occurred repeatedly in the independent evolution of multiple clades of long-lived animals from shorter-lived ancestors: the progenitors of bats, ${ }^{2}$ birds, flying squirrels, elephants, turtles, chimps and island-bound opossums ${ }^{3}$ have all managed this trick when they found themselves in ecological niches in which delayed reproduction could pay off in Darwinian fitness. Evolution, constructing a new form, does not start with a blank page but rather morphs by pulling a minimum of well connected strings. Turning a wolf into a Chihuahua or a mastiff, or a hamster brain into a monkey brain, ${ }^{4}$ does not involve mutations in large sets of genes of similar purpose (the gene for shorter leg bones, the gene for shorter jaw, the gene for shorter esophagus. ..) but instead must involve mutations in a small number of loci that act to control, directly or indirectly, a wide range of interconnected downstream processes. In the gerontological context, a new allele that postponed tumor development (or atherosclerosis or cataracts or osteoporosis) for 40 years in a short-lived primate would have little survival value on a genetic background that permitted fatal declines in muscle or immune strength in the first decade of life. Genes that slow down the rate of degeneration of only a single physiological system are not likely to have much effect on reproduction or survival at advanced ages; only mutations at loci that slow down the rate of change of a wide range of senescent processes are likely to have much survival value in species that find themselves in low risk niches that select for longevity. Thus, the idea that aging involves a concurrence of multiple processes, each under independent genetic control, that just happen to all occur much more slowly in people than in dogs, and more slowly still in mice, seems improbable.

The caloric restriction (CR) phenomenon also highlights the implausibility of the multiple process view of aging. Nearly all of the effects of aging, including changes in gene expression, cell communication, extracellular matrices, and disease risks, can be retarded in parallel by caloric restriction of laboratory rodents. ${ }^{5}$ Although the mechanism of CR is still unknown, it is hard to believe that a unimodal intervention of this kind would, by a bizarre coincidence, retard such a wide range of age-sensitive processes unless the rates of these processes were themselves at least partly coupled.

The tension between the concept of aging as a unitary process and the idea of aging as a set of distinct processes can perhaps be clarified by an analogy. In some ways an aging organism might be modeled as a complex system of water pipes, connected by a system of valves and side channels, with fluid entering from a reservoir at the top and leaving through various taps and drainspouts. Differences among individuals in the pattern of flow could be influenced by many factors, including the diameter of the individual pipes and connectors, the set point of the various valves, and the activities of sensors that regulate flow in distant channels based upon flow, or turbulence, or acceleration of liquid in monitored pipes. This plumbing system, like an aging organism, has many interdependent yet distinct processes in action simultaneously, each of which may have some influence on the time at which the supply cistern becomes exhausted. An evolutionary process, or an intervention like caloric restriction, might extend the lifespan of the system by tightening each of the values and narrowing each of the tubes proportionately and simultaneously - a tricky approach - or might accomplish the same thing more simply by replacing the water with glycerol. The single process model of mammalian aging can be seen as a search for the factors regulating the viscosity of the fluid coursing through the multiple channels of life history, and the speciation and caloric restriction arguments can be taken as encouraging hints that the factors may be manageably few in number.

Where, then, does it now seem worthwhile to look for the biochemical basis of the aging process? The remainder of this essay will consider several proposed answers, each with its advocates, none yet wholly successful. We will consider, in turn, problems and prospects in the areas of comparative biology, mammalian and invertebrate genetics, biomarker research, caloric restriction, and clonal senescence, and then conclude with a discussion of potential links berween aging and late life disease.

\section{COMPARATIVE AND EVOLUTIONARY BIOLOGY}

In one sense the comparative biologists, with their cousins the quantitative geneticists, have already solved the problem of aging by producing coherent, largely confirmable explanations of how evolutionary forces regulate lifespan and other life history parameters. Details of this argument, traceable to ideas of Peter Medawar and George Williams, quantified by Charlesworth and his students, and supported by laboratory ${ }^{7,8}$ and field ${ }^{3}$ data, are beyond the scope of this paper but can be sketched out in summary form. Consider a hypothetical species in which aging does not occur. Even in the absence of aging, the mortality rate cannot be zero; some factors, such as disease, predation, and bolts of lightening, will kill off some members of the population in any given period of time. In fact, with such a constant (and ageindependent) risk of mortality, the survival of any given cohort will resemble the exponential decay curve of a given mass of a radioactive element. If for a particular species in a particular locale the risks are such that $50 \%$ of the population die each year, then $25 \%$ of a given cohort will be alive at the end of the second year, and $12.5 \%$ at the end of the third year. In such a situation, a mutation that increases the risk of a particular disease - such as cancer, or atherosclerosis, or immunosenescence leading to fatal influenza - in 1-year-old animals will be strongly selected against, because there are many 1-year-old animals, each with an appreciable probability of contributing offspring to the next generation. A mutation that causes the same illnesses, but does so only in 8-year-old animals, will, however, not be weeded out by the 
forces of natural selection, because natural attrition has whittled the pool of 8-year-old animals to a negligible remnant, still by hypothesis healthy and fertile and no more likely to die this year than they were last year, but so few in number as to contribute very few genes to the next generation. Thus, genetic variations that cause disease only at older ages tend to accumulate. Mutations that improve survival or fertility at early ages (say the first 2 years in this example) will be strongly selected for, even if, as a pleiotropic side effect, they lead to illness and diminished fitness at later ages. These two factors, accumulation of harmful late-acting mutations and of mutations whose early beneficial effects are accompanied by later deleterious effects, generate an animal in which the chance of disease and physiological decrepitude become increasingly more likely at older ages - in other words, an animal that ages.

Now modify the environment. Remove the owls, install lightening rods, and kill off the mosquitoes that spread the infections. Under these new conditions survival to age 8 , once very rare, becomes more likely, and alleles that cause degenerative changes at this age are for the first time deleterious, and, thus, subject to evolutionary pressures. New alleles that postpone cancer, vascular disease, loss of reflexes, diabetes, and musculoskeletal frailty until the age of 20 are suddenly highly beneficial. The key prediction of this model, now confirmed by a variety of descriptive data and even some experimental evidence, is thus that environments where baseline risks are relatively low permit the evolution of species (or populations) in which senescent changes are also delayed. Species on predator-free islands, species that are big enough or have tough enough carapaces not to get eaten, mammals that learn to fly or glide- each of these conditions tends to evolve species or races with the potential for a long lifespan.

The challenge now is to convert this satisfyingly complete, quantitative, explanatory framework into a set of new animal models for tackling the important questions of mechanistic gerontology. There is a substantial tradition within aging research of trying to use comparative biology to address questions in cellular and molecular biology, but these efforts have too often suffered from a lack of the professional perspective that a sophisticated comparative biologist might have brought to the project. ${ }^{9,10}$ Plots of longevity against specific biochemical or cellular parameters, such as fibroblast expansion limits or cytochrome levels or DNA repair capabilities, have from time to time suggested a relationship between the trait of interest and species-specific longevity, but they suffer from an arbitrary and often ill-considered selection of species chosen for their familiarity rather than on the basis of evolutionary relationships. Plots of fibroblast expansion limits versus species longevity can, for example, be generated with positive, negative, or level slopes, depending on selection of species and adjustment for body size (see Figure 1). Comparison of outbred white-footed mice (genus Peromyscus) to inbred laboratory mice (genus Mus) may be tempting, based on superficial similarities, but such hasty comparisons overlook the rather distant evolutionary relationship between these two species as well as the confounding influences of laboratory adaptation and inbreeding. Claims that the remarkably long lives of bats reflect merely the effects of hibernation can be overturned simply by looking up the lifespans of bats that do not hibernate ${ }^{2}$; claims that the ratio of brain weight to body weight provides a useful predictor of lifespan among species seem less remarkable when one notes
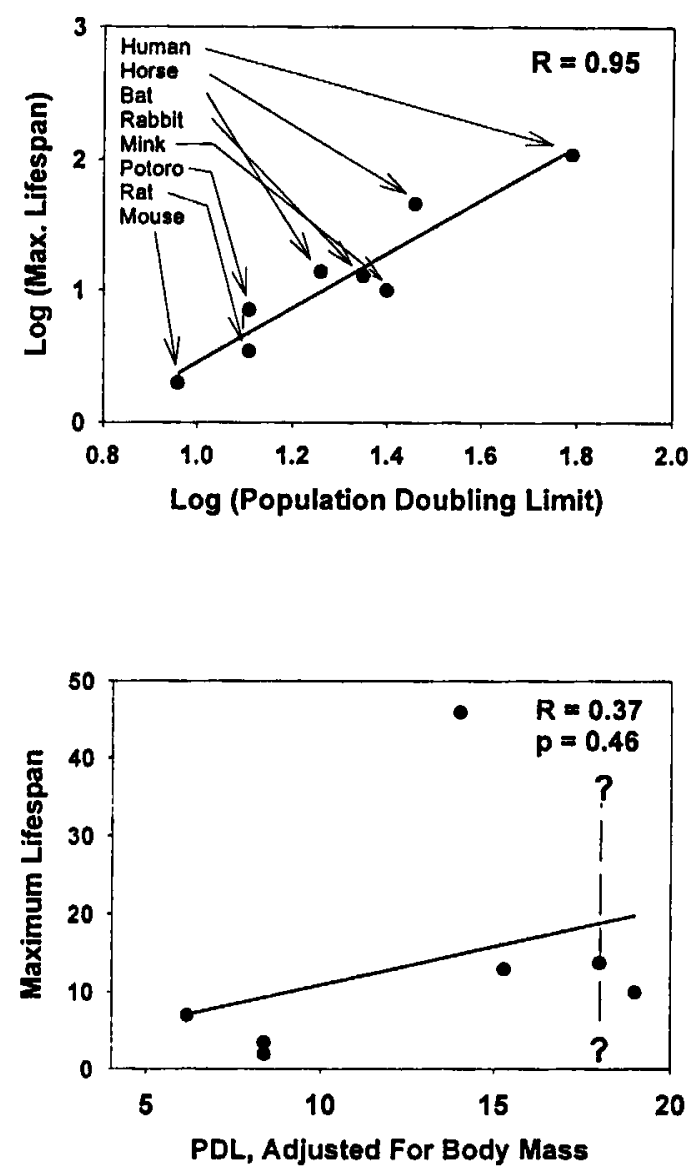

Figure 1. Two plots showing inter-species comparisons of fibroblast culture data with species longevity. The top panel shows data from reference (58); fibroblasts were cultured until growth cessation, and the logarithm of the number of population doublings was plotted against the logarithm of the maximal longevity for the species. The correlation is highly significant. The bottom figure shows these data after adjustment for differences in the number of doublings needed to account for differences in body size relative to the smallest species (bat). Thus for example, the population doubling value for horses was reduced by 28.8 to 14 to reflect the need for 14.8 cell doublings to create a horsesized body from a bat-sized body. With this adjustment the strenght of the correlation $(R=.91, P=.001)$ is determined almost entirely by the values for humans, which exhibit both extreme longevity and extreme growth potential. Exclusion of the single point for human data yields the curve shown in the figure, where $R=.37, P=.46$. The dashed line with question marks indicates that the lifespan for the bat species used in reference $(58)$ is unknown; other bats in this subfamily $(N=38)$ have maximum documented lifespan ranging from $S$ to 32 years. This result suggests that the claimed relationship between fibroblast growth potential and species longevity largely reflects the dependence of both measurements on body size, together with the atypical properties of human cells. The bottom panel and associated calculations were provided by Dr. Steven Austad.

that ratios of liver, heart, spleen, or kidney weight to body weight are even better predictors. ${ }^{11}$

Thus the comparative tradition in biogerontology has a checkered past. It is not hard, however, to imagine ways in which the comparative perspective might be used to develop new models to test ideas about the aging process. Ideas about the importance of oxidative damage in the aging process 
might benefit from studies of bird species that combine high rates of fuel utilization with long lifespan. ${ }^{12}$ Island populations, which can be long-lived in comparison with closely related mainland populations, ${ }^{3}$ may also provide useful test systems for examining ideas about the way in which aging rate is controlled. Genetic crosses between long-lived island populations and their shorter-lived cousins may be very helpful in defining the numbers, positions, and effects of longevity-regulating loci. Field experiments in which fish are transferred from high predation to low predation conditions ${ }^{13}$ can very quickly produce new populations that differ in maturation rate and body size; if further study showed that such populations also differed in aging rate, they (or mammalian analogues) could provide exceptionally valuable resources for the genetic and biochemical dissection of aging and its relation to late life diseases. Meeting this challenge will require courage, imagination, and careful planning on the part of both bench scientists and those who fund them and provide long-term support for development of new models.

\section{MUTANTS, INDUCED AND NATURAL}

The study of mutants, whether spontaneous or induced by transgenic and knockout technology, is certain to play an increasingly important role in reductionist gerontology and can be seen as a special case of comparative biology. The discovery of alleles that extend lifespan in invertebrates by three-fold or more, ${ }^{14,15}$ the creation of transgenic flies in which altered antioxidant defenses seem to extend lifespan, ${ }^{16}$ and the cloning of the mutant gene for Werner's syndrome, which seems to accelerate a subset of the physiological and pathological changes often seen in aged people, ${ }^{17}$ all have earned widespread attention recently as important steps towards improved understanding of the biology of aging. It is tempting to overinterpret findings in this genre, and the caricature of Werner's syndrome as a straightforward acceleration of the aging process, though carefully avoided by most of the researchers involved in the analysis of WRN mutants, has proven irresistible for many popularizers despite the many differences, some of them very obvious, between Werner's patients and normal older individuals. Mutations that shorten the lifespan, such as the misnamed "senescence-accelerated mouse" lines, ${ }^{18}$ must always be viewed with great suspicion as models of aging because there are many ways to impair viability without interfering with aging per se, but mechanistic analysis of syndromes such as Werner's, that appear to produce collections of traits that are rarely seen together except in the aged, seems likely to provide new insights into aging not otherwise easily obtained. The mere existence of single gene mutations that lead to dramatic increases in longevity demands intellectual gyrations ${ }^{19}$ for those who prefer to consider aging as a collection of independent processes that just happen to occur in rough synchrony in any one species since single alleles that, by chance, happen to retard all such processes to a similar extent seem unlikely to arise.

Evaluation of claims for single gene retardation of aging requires careful assessment of the control strain(s) to which the mutant is compared. A recent publication has demonstrated ${ }^{20}$ extended lifespan in mice bearing the mutation $\mathrm{df} / \mathrm{df}$, which leads to small size by inhibiting the development of growth hormone-producing pituitary cells, and other dwarf mice, such as the dw/dw homozygote, also seem remarkably long lived (K. Flurkey and D. Harrison, unpub- lished results). These reports, the first published evidence for single gene effects that extend lifespan in a mammal, could indicate important interactions between the aging process and the factors that control size in mammals, an interaction made more attractive by the negative correlations seen between size and longevity in $\operatorname{dogs}^{21}$ and, perhaps, other mammals. Caution is called for, however, until data become available to see if the effects of the $d w$ and df alleles are also seen on a variety of genetic backgrounds; if these effects are seen only on the specific genetic backgrounds tested so far, their implications for theories of aging become more tenuous. Similarly, reports that transgenic flies exhibit extended lifes$\operatorname{pan}^{16}$ are somewhat tempered by the realization that the extension is only in comparison with specific, short-lived control strains rather than in comparison with the lifespan of unmanipulated flies. These reservations may, one hopes, be merely temporary, and the increasing sophistication by which synthetic geneticists can now manufacture mice in which specific genes can be ablated, expressed at graded doses, or turned on or off at specific times is certain to be a major influence in experimental gerontology for the foreseeable future.

\section{INVERTEBRATE GENETICS}

The scientists who study aging in nematode worms (Caenorhabditis elegans) and fruit flies (Drosophila melanogaster) do so because of the tremendous power these two species provide for genetic analysis and genetic manipulation; but these researchers are well aware that neither species is a mammal. There is no particular reason to suspect that the processes that limit life in these two species have major roles to play in mammalian aging. There is, however, also no compelling reason to conclude that mammalian aging is so unlike invertebrate aging as to make the latter field certifiably useless. The processes that plague old people - atherosclerosis, Alzheimer's disease, immune senescence, various cancers - surely play no role in fly or worm mortality, whereas drowning in mashed bananas as a consequence of wing erosion is, conversely, a rare cause of death in mice and humans. But the cause of aging, as opposed to the cause of death, is, at this point, perfectly mysterious in all of these species. Thus an assessment of whether specific processes, such as oxidation damage or mitochondrial dysfunction or gene dysregulation, play an important role in both human aging and aging of one or both invertebrate models can only be a matter of educated intuition, guesswork, or faith. Many fundamental discoveries about mammalian development have, to date, emerged from clues provided initially by studies of C. elegans and/or D. melanogaster that it would be foolish to bet too heavily against the invertebrate models.

The proof of this pudding, the future landmark study, may come from attempts to locate and analyze mammalian homologues of longevity-extending genes initially found in flies or worms. Recent work in worm genetics has produced at least two remarkable breakthroughs although implications for mammalian aging are still uncertain. First, several groups $s^{14,15}$ have contributed to the demonstration that adult worms have a genetic pathway, normally silent in adult life, that when activated by mutation can extend lifespan by three-fold or more. The details of this pathway are now under intensive study; once the key players are cloned, it will be possible to formulate and test hypotheses about their mode of action and to seek for mammalian genes of similar sequence 
and, possibly, related effects. Second, activation of this lifespan-extending pathway by mutations in several different genes seems to increase the resistance of the worms to a variety of stresses including oxidation, UV damage, and thermal stress. ${ }^{22}$ It is certainly plausible, though not yet quite proven, that the lifespan effects of the mutations are themselves mediated by the changes in stress resistance. If this idea proves correct, it will suggest experiments to alter aging rates, and consequently disease incidence, in mice or eventually, perhaps, in humans.

\section{BIOMARKER RESEARCH}

The key goal of biomarker research, a goal still very far from realization, is to develop a set of tests that can be performed, fairly early in life on either mice or humans, that can be used to identify individuals who are aging at different rates. Some authorities argue $e^{2.3}$ that such a goal is nonsensical, because the consequences of aging are uncoordinated, with each individual developing his or her own unique phenotype in old age. In this view, assigning a single number as a "measure of biological age" is as hopeless an assignment as trying to quantitate beauty or virtue or talent along a single dimension. Others, myself among them, take a more optimistic perspective, but we must admit that no set of well validated biomarker assays has yet been developed for mammals. The presence of confounding factors poses a major technical obstacle: even if, for example, measurement of $T$ cell function or muscle endurance or nerve conduction time or collagen cross-linking could provide some indication of interindividual differences in the hypothetical "rate of aging," each of these and other similar candidate measures are also strongly influenced by a range of other influences, most of the them poorly understood. T cell subsets, some of them robustly age-sensitive in both humans and mice, are also influenced by genetic polymorphisms, by chronic and acute stresses, by irradiation, by medication, and by infectious agents. To discern in all of this "noise" the tiny bit of effect hypothetically produced by individual variations in aging rates is difficult, and it might be impossible.

Merely showing that a given assay changes with age, and thus distinguishes most old people from most young people, is not sufficient to qualify a test as a biomarker. What counts is showing that the test in question divides people (or mice) of a given age into groups that differ predictably in a wide range of other age-sensitive traits. Among the possible biomarkers, risk factors for mortality have a special status; many of them are already well defined for humans and are of special interest to physicians and insurers. Risk factors for death may or may not eventually prove useful as biomarkers of aging. Some risk factors, such as smoking history, propensity for seat belt use, and having recently been taken captive in a prison riot, are clearly associated with increased mortality risk but do not seem likely to serve well as indices of aging. Other factors, such as genes predisposing to Alzheimer's disease or myocardial infarction, raise all-cause mortality risks by effects on specific common illnesses but, again, are probably not useful monitors of an underlying aging process with effects on a wide spectrum of lethal and nonlethal late-life changes. Showing that an assay not only predicts subsequent mortality but also correlates in plausible ways with other age-sensitive traits is a minimal standard to which proposed biomarkers must be held (see Figure 2 for an example of such an approach).
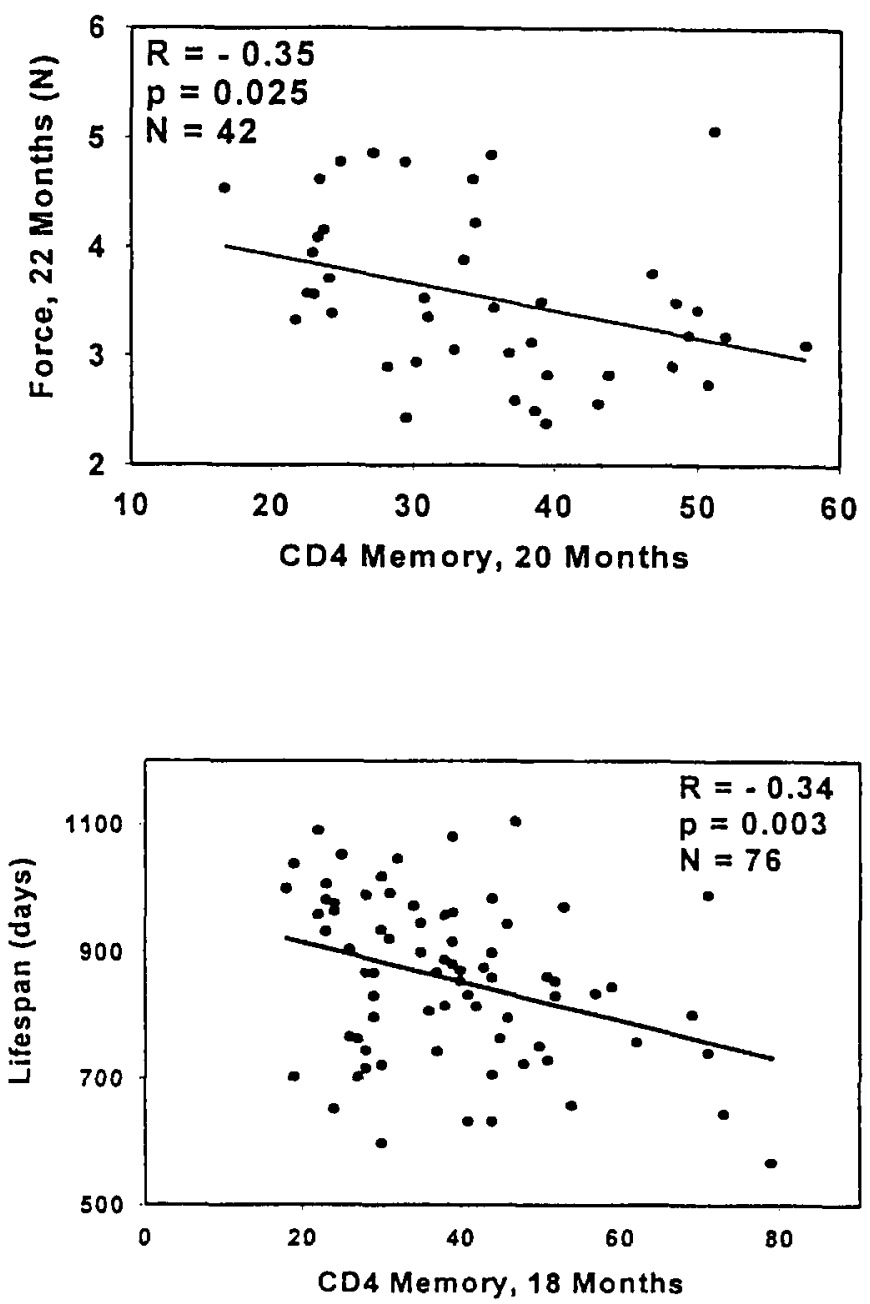

Figure 2. Biomarker validation: a mouse example. The top panel shows results of a study of 42 male and female genetically heterogeneous mice (Faulkner, van der Meulen and Miller, unpublished data). Each mouse was tested at 20 months of age to determine the fraction of the peripheral blood CD4 T cell subset that expressed the surface markers of memory $T$ cells; this trait typically increases with age. Each mouse was tested at 22 months of age for muscle force generation; this trait typically declines with age. The negative correlation suggests that mice with high CD4 memory cells ("old" immune systems) also have poor force generation ("old" muscles). The bottom panel shows results from a separate study of 76 female mice from the same genetically heterogeneous stock. Mice that had high levels of CD4 memory cells at 18 months of age were found to have significantly lower remaining life expectancy than siblings with lower levels of CD4 memory $T$ cells at the same age. Taken together, these results suggest that $C D 4$ memory $T$ cells might be able to serve as a biomarker of aging in mice.

Comprehensive, well validated sets of innocuous tests of individual age would be of very great value for gerontological research, in the same way that the invention of the sphygmomanometer served as a critical step in hypertension research. On a theoretical plane, such tests would provide a major pillar of support for the single process model of aging. They would provide a useful stimulus to mechanistic biogerontology, challenging molecular and cellular scientists to find the hidden links connecting diverse biomarkers to a central pacemaker. In the practical sphere, sets of biomarkers would 
facilitate short-term tests of antigeric interventions. The development of a set of useful biomarkers of aging in monkeys, from a much larger set of age-sensitive variables tested for their reproducibility and their ability to track longitudinal changes in individual animals, has already started to demonstrate the potential power of the biomarker approach ${ }^{24}$ through an analysis of blood antioxidant levels as modulators of aging rate and disease risks. Extension of this pioneering work to more tractable or more self-conscious species (mice and humans, respectively) will deserve landmark status, if it can be done at all.

\section{CALORIC RESTRICTION}

It has been known for more than 50 years that rodents, if fed a nutritionally complete diet that contains about $40 \%$ fewer calories than they would choose to consume given free access to food, live up to $50 \%$ longer than rats or mice on an ad libitum diet. This lifespan extension, a robust phenomenon that has now been repeated in dozens of laboratories using many different strains of mice and rats, does not merely represent a retardation of one or a small group of lethal diseases but seems to involve a genuine deceleration of the aging process as a whole in that a very wide range of biochemical, genetic, and physiological concomitants of aging seem to be retarded in parallel. ${ }^{5,25}$ Although the largest effects are seen when the restricted diet is begun early in life, restriction extends lifespan even when imposed in middle-age. ${ }^{26}$ Both mean and maximal lifespan are affected. Many early ideas about the mechanism of the caloric restriction (CR) effect have been refuted, including the suggestions that $C R$ worked by retardation of growth, by reducing the ingestion of toxic substances, ${ }^{27}$ or by lowering the amount of energy utilization per gram of metabolizing tissue. ${ }^{28}$ The idea that the CR protocol merely restores "normal" levels of food intake, i.e., those an animal would consume in the wild, seems inconsistent with the observation that restricted rats, unlike rats in nature, are largely infertile. ${ }^{29}$

One obvious challenge is to discover how $\mathrm{CR}$ works to retard so many aspects of biological aging, including the age-adjusted incidence rates of almost all the common latelife diseases seen in rodents. Many plausible suggestions are based on well documented effects of CR, including diminished blood glucose and insulin levels, ${ }^{30}$ increases in free glucocorticoid levels, ${ }^{31}$ altered expression of heat shock genes, ${ }^{32}$ and changes in resistance to free radical-mediated damage. ${ }^{33}$ The difficulty is to discover which of these alterations are primary, which are secondary but mechanistically critical, and which are merely incidental. Increased emphasis on the initial effects of CR may be helpful here, by discriminating between changes that "merely" represent the deceleration of changes normally seen in aging animals and those that are seen relatively early after imposition of the CR diet and not typically affected by aging per se. The latter class of phenomena is likely to include some of the primary effects by which CR works its wonders but will also include CRdependent effects that are independent of its interactions with aging. CR mice, for example, may have relatively low levels of muscle force generation, not because this is characteristic of young animals, but because CR mice are small, with correspondingly diminished muscle cross-sections.

Showing that $\mathrm{CR}$ retards aging in primates will also qualify for landmark status. At least three studies are already underway that could help to settle this important ques- $\operatorname{tion}^{34-36}$; each has shown some mildly encouraging preliminary results, but none has settled the issue, nor can a speedy answer be expected from lifespan studies of such long-lived animals. An approach based on biomarkers would clearly be helpful in primate studies, and validation of biomarkers in monkeys is making excellent progress, ${ }^{24}$ but researchers will need to beware of the tempting, but fallaciously circular, assumption that interventions that alter candidate biomarkers must necessarily be influencing the aging process. Studies of inter-individual variation in the efficiency of fuel utilization may also lend themselves to use in studies of primates, including humans: people who, like CR rodents, maintain low glucose and insulin levels while converting a high proportion of fuel to work might in principle constitute a subset whose metabolism already resembles those of restricted animals.

A detailed mechanistic understanding of caloric restriction could have dramatic implications for disease prevention. The extension of healthy lifespan already achieved in laboratory animals by the CR method is proportionally greater than that which would be achieved in humans by the combined elimination of all cardiovascular disease, cancer, and diabetes. ${ }^{37}$ Furthermore, CR rodents are not frail and debilitated for an extended period at the end of the lifespan. In fact, they are remarkably robust; given access to a running wheel, for example, they spontaneously run several kilometers/day at ages at which nearly all control animals have long since died (Figure 3). A method to reproduce the CR effect pharmacologically would be worth a pretty penny and seems no less difficult to develop than, say, a cure for breast cancer or Alzheimer's disease. Developing methods to reproduce some of the key secondary effects of the CR protocol, such as alterations in glucocorticoid levels or in glucose/insulin ratios, might also deserve attention, initially in animal models and eventually in clinical trials.

Running Activity

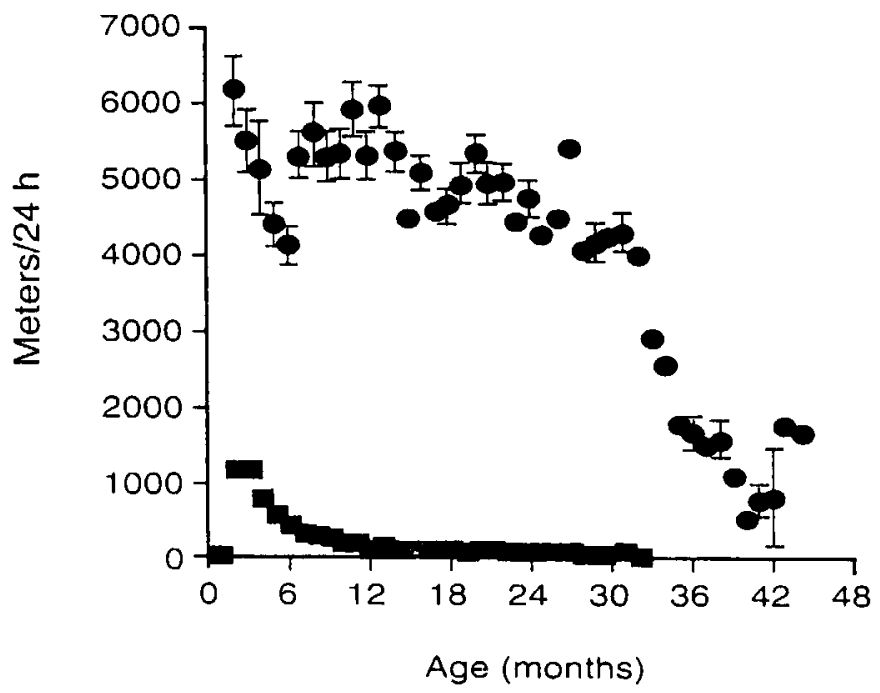

Figure 3. Spontaneous exercise activity by rats on a restricted calorie diet. Control rats (squares) given free access to an exercise wheel run approximately $1 \mathrm{~km} / \mathrm{day}$ initially, but this activity declines to less than $200 \mathrm{~m} /$ day by the age of 12 months. In contrast, calorie-restricted rats (circles) average more than 4 $\mathrm{km} /$ day until more than 30 months of age and are still running more than $1 \mathrm{~km} / \mathrm{day}$ at ages at which nearly all of the control animals have died. Figure courtesy of Dr. Roger McCarter. ${ }^{59}$ 


\section{VERTEBRATE GENETICS.}

Genetic experiments that take months to perform in flies can take years or decades to carry out in mice, and would, if ethically permissible, take centuries to attempt in humans. The attraction of mouse genetics for experimental gerontology thus arises not from its convenience or simplicity but from the plausible guess that mouse aging and human aging are as closely related as the anatomy and cell biology of these two species: i.e., not awfully close, but maybe close enough to do the trick. The key unanswered question here is whether the number of genes controlling aging rate are few enough that each one may have an effect large enough to be detected in studies cheap enough to be practical. This question, simple to pose, proves to be hard to answer with confidence on current evidence. It is clear that there are thousands of genes at which mutations can affect lifespan, usually by leading to a lifeshortening disease. The trickier question is whether some loci also affect lifespan by a general effect on the aging process. Such a locus would be identifiable if it affected not merely the likelihood of a single lethal illness, or a small number of related illnesses (e.g., a variety of lymphomas) but instead altered the incidence of most or all late-life illnesses and, at the same time, altered the pace of a wide range of agesensitive changes. The questions to be answered, in rough order, are three: how many such loci are there, where are they located, and what do they do? There are hints ${ }^{38,39}$ that major differences in longevity can be brought about by differences in as few as 3 to 12 genes (or, more precisely, groups of closely linked genes), but these studies are still fragmentary and not fully convincing. The recent availability of easily scored genetic markers that discriminate among various mouse strains has made possible an approach, called quantitative trait locus (QTL) mapping, that may, in the next few years, provide the first compelling data on the location of mouse genes that influence longevity.

Success of the QTL approach would have important long-term and short-term consequences for biomedical gerontology. In the short term, it would be important to see the extent to which the implicated chromosomal regions also had effects on multiple aspects of the aging process. If, for example, one or more of the genetic markers found by QTL mapping were also found to influence the rate of decline of immune function, muscle strength, and wound healing in aging mice, this would lend powerful support to the "single process" models of aging, and spur interest reciprocally in the QTL loci themselves. Mice differing at one or several QTL could also be used to test specific mechanistic ideas about the control of aging: if a group of mice selected for a specific set of QTL were found to live long and healthy lives, but not to differ from their sibs in, say, antioxidant defenses, this would provide potent evidence against the free radical theories of aging. Strong evidence that one or more markers was linked to loci that influenced a wide range of age-dependent changes would provide a rationale for the very difficult work needed to identify and clone the effective genes themselves. Studies of the effects of segregating portions of the homologous human chromosomal regions could be initiated well before the cloning of the mouse genes themselves and might help to test the usefulness of the mouse genetic systems as decent models for the human genetics of aging. Success in any of these projects would merit landmark status and help to put experimental gerontology on a rational footing.
The QTL approach, though, is likely to fail for one of two reasons. It may be that there are too many loci at which variations contribute to individual differences in aging rate; if so, the contribution of any one such locus would be too small to detect. An alternate, and less intractable, reason for failure might reflect a peculiarity of the world's pool of laboratory mice: their minuscule stock of genetic variation, particularly at loci that might in principle have an effect on aging. Like cheetahs, ${ }^{40}$ nearly all laboratory mice are derived from a very small number of ancestral animals. This "founder" effect might by itself have accidentally rid the laboratory mouse gene pool of variation at the hypothetical age-controlling alleles. Worse still, the very strong and unintended selection that accompanies adaptation to laboratory life - selection for tameness, for rapid sexual maturation and large litters, and for loss of loci that coordinate life history to seasonal variations ${ }^{41}$ and periods of food scarcity - create a gene pool that is radically altered and altered in ways that may select against high longevity alleles. In the evolutionary context-high predation pressure leading to rapid development and rapid aging - the laboratory scientist serves most laboratory mice as predator extraordinaire, ruthlessly selecting against genotypes that delay reproduction. This potential obstacle, however, can potentially be overcome, by starting the genetic analysis with mice freshly trapped from wild populations, preferably from island stocks in which natural selection may already have selected for genotypes with delayed maturation and delayed senescence.

\section{CELLULAR SENESCENCE}

If the educated layperson (a first year medical student, for example) knows anything about the experimental biology of aging, he/she knows that normal cells can't continue to divide forever. The report ${ }^{42}$ that human diploid fibroblast cultures are limited in the number of cell divisions they can accomplish before ceasing to replicate has entranced a generation of cellular gerontologists. The intuition that this cell culture system is in some sense a model for aging of intact organisms is viewed as whimsical by skeptics, but even the skeptical acknowledge that study of the "Hayflick limit" continues to motivate high quality research on a variety of major problems in cell growth control and differentiation. ${ }^{43-46}$ Ten years ago the principal challenge for the students of in vitro senescence was to work out the molecular basis for growth cessation in late passage cells; this important problem, though not yet fully solved, has clearly begun to yield, and it is now reasonably easy to see the outline of the eventual solution. The new challenge, still only tentatively faced, will be to determine whether the phenomenon of in vitro senescence bears an important relationship to, or sheds new light on, the aging process that turns teen-agers into geriatric cases.

One important goal will be to determine if cellular senescence occurs in vivo and, if so, at what ages and in which tissues of what organisms. One obstacle has been the lack of unambiguous single-cell assays that can distinguish latepassage cells from their early-passage progenitors and, thus, serve plausibly as an marker for senescent cells in intact organisms. One promising report ${ }^{47}$ has shown that skin of older (but not younger) persons includes a small proportion of fibroblasts and keratinocytes that express a $\beta$-galactosidase isoform characteristically seen in late-passage fibroblast cultures. Extension of this approach to the analysis of other 
cell types in other tissues, and development of other similar markers, including some suitable for use in rodent studies, will be very helpful in testing hypotheses about the presence of cells, in older individuals, whose properties resemble those seen in late passage cell cultures. New ideas and new approaches are also needed to address the related issue of whether senescent cells, if they are present at all in tissues of aged subjects, contribute at all to disease pathogenesis and organ dysfunction. In this context it may be helpful to redirect attention away from the final stages in the course of in vitro senescence, i.e., those related to growth cessation, to changes that occur in middle-passage cultures, inasmuch as cells at these intermediate stages may prove to be more common in the tissues of aging individuals. Other properties of senescent fibroblasts, such as secretion of collagenase ${ }^{48}$ or resistance to apoptosis, ${ }^{49}$ may prove to be particularly important as mediators of pathophysiological changes in old age.

The idea that the length of telomeres (the terminal segments of chromosomes) may provide a way of counting numbers of cell divisions has led to studies showing a trend towards shorter telomeres both in late passage cultures and in some cell types of aging people, ${ }^{46,50,51}$ and there are some data, preliminary but provocative, to suggest that short telomere lengths may be characteristic of endothelial cells in atherosclerosis-prone regions of the vascular tree..$^{52}$ Early, influential, models for age-dependent changes in telomere length depicted the trajectory of telomere length, like the aging process, as essentially a one-way street, in which the silencing of the enzyme telomerase in early embryogenesis prevented further increases in telomere lengths, which then shortened progressively at each cell division because of well documented properties of the polymerase that synthesizes DNA. ${ }^{53}$ Over-short telomeres were postulated to promote neoplastic transformation via chromosomal rearrangements and to lead to cell growth cessation by processes still to be elucidated. Activation of telomerase was seen as a key step in the development of malignant tumors, enabling them to overcome the barrier to indefinite growth that would otherwise be imposed by the Hayflick limit. ${ }^{54}$ This appealing picture has been complicated recently by demonstrations that telomerase activity can be detected in at least some normal cells ${ }^{5.5}$ and that telomere length in neoplastic cells can be modulated by telomere-binding factors ${ }^{56}$ that block telomerase function, while the role of telomere shortening in growth cessation at late passages has also been questioned. ${ }^{57} \mathrm{~A}$ thorough understanding of the role of cellular senescence as a barrier to malignancy, and the possible role of telomere length in both processes, might help to clarify the nature of the linkage between cancer, aging, and species-specific maximum lifespan.

The ability of aging to alter the properties of cells that are unable to divide in adult life has always posed a stumbling block for devotees of systems for studying growth cessation in late passage cells and for their latter-day cousins the telomere specialists. While it is possible to devise possible connections between a loss of cell proliferative function and secondary changes in nonproliferative cells and extracellular tissues, attempts to test these possible links are still at a very early stage. The difficulty of concocting a theory that explains the parallel loss of adaptive function in so many cell and tissue types is, of course, a major obstacle for most theories of aging, not only for those that focus on changes in cellular proliferation.

\section{LINKS TO DISEASE}

One way to put the strength of the links between aging and disease into perspective is to note that the risk, each day, of a single cell undergoing the transformation that develops into a fatal neoplasia is about 100,000-fold lower in humans than in mice. The cancer risk in humans, i.e., the risk of first noticing a cancer that if left untreated would be fatal, is about $.42 \%$ per year, while the corresponding risk in mice is about $20 \%$ per year, a 50 -fold difference. This contrast, already dramatic, is magnified still further by the difference in the size of the target population. Most cancers are clonal in origin, and a 10 -fold increase in cell number ought, all things being equal, to lead to a 10 -fold increase in the likelihood that any one cell will accomplish each of the steps that convert normal to malignant cells. The 2000 -fold increase in cell size that produced $70-\mathrm{kg}$ humans from some $35-\mathrm{gm}$ mouse-like ancestor should thus have led to a 2000 -fold increase in cancer risk per animal per day, not the 50-fold decrease that is, in fact, observed. Evolution, in producing long-lived big animals, needs somehow to build in a defense system that reduces per cell malignancy rates by five orders of magnitude. Merely reducing the rate of one kind of cancer, say mammary adenocarcinoma, by 100,000 fold would not be enough because the resulting large animal, with incidence risks of all other cancers left at the "mouse-normal" rate, would be virtually certain to die before age 5 to 10 of some other tumor type, perhaps lymphoma or hepatoma. And merely reducing by 100,000 fold the rates for all forms of neoplastic disease would also be useless unless evolution managed simultaneously to prevent the decline in immunity, muscle strength, wound healing, and neural responsiveness that would greatly impair survival in mice that managed to avoid cancers by 5 or 10 years of age. A cure for aging, something that evolution has managed to accomplish many times in the development of long-lived, large animals in multiple mammalian lineages, and which gerontologists have been able to mimic (partially) for decades by caloric restriction in rodents, is effectively a cure for disease.

Developing a cure for aging might take a while; in the meantime, experimental pathologists can turn their attention to the related problem of discovering the mechanisms that link specific diseases of interest to the aging process. The challenge proposed here is not the familiar (and noble) one of working out the cause of specific diseases, the causes of cancer and atherosclerosis and sarcopenia and immunosenescence, but instead to learn more about why these and other maladies are postponed so dramatically in humans, bats, and some birds and correspondingly accelerated in mice, opossums, and other short-lived mammals. Studies of closely related species, races, and subspecies, chihuahuas versus wolfhounds, ${ }^{21}$ mainland versus island opossums, ${ }^{3}$ humans versus rhesus versus lemurs, ${ }^{11}$ and species that have similar sizes but radically different lifespans, ${ }^{12}$ may be particularly germane. Well specified mechanistic theories (e.g., "the 10fold lower cancer risk in the 8-year-old chihuahua, compared with similarly aged wolfhounds, results from improved efficiency of repair of DNA damage throughout the adult lifespan") can be tested and either discarded or, if supported by the evidence, tested again in other comparative contexts. Comparisons within species may also be informative; it would be interesting to know if the genetic factors that lead to similar longevity in identical twins ${ }^{12}$ or, to a lesser extent, 
among first degree relatives, also influence such factors as immune function, muscle function, insulin sensitivity, DNA repair, or other traits of interest. Synthetic genetics may also provide useful leverage; it would be interesting to know, for example, if mice engineered for improved protection against free radicals, or selected for high levels of immune responsiveness, ${ }^{38}$ show parallel retardation in a number of agedependent processes, including risks of a variety of diseases.

\section{SUMMARY}

When will the biology of aging become useful? Doing basic science requires a leap of faith. The trapeze artist, flinging herself backwards off her perch, makes the reasonable assumption that her partner will once again swoop into the spotlight in the nick of time to prevent the embarrassment of a missed connection, and she is seldom disappointed. The basic scientist, similarly vulnerable, arguing the case for basic research against the claims of others who maintain that the money could be more profitably spent on problems whose solutions are easier to envision, must try to avoid looking down to the circus ring below, filled as it is with the mangled remains of basic research ideas whose uselessness has already been amply proven. Is there any way to improve the chances that the direction in which we hurl our careers (and the hard-earned dollars of our sponsors) will indeed contain something to grab at the other end of the arc? Our colleagues, hard at work learning how to improve the well-being of the seriously ill, or adding a methyl group here and there to drugs that already work somewhat, or delineating the detailed pathogenesis of common diseases whose mechanisms are already fairly well understood, can feel justifiably proud of their own accomplishments and confident that the future will hold more along the same lines. They have earned the right to wonder when, if ever, studies of the biology of aging will come of age. Those of us who urge increased support for studies of the fundamental biology of aging can recite lists of basic science that did indeed make the big time - studies of DNA-cutting enzymes, of subatomic particles, of mutant fruit flies, of game theory - but in our hearts we know that this bravado cannot substitute for proof that we know something important about how aging works and can do something with that knowledge.

The most valuable landmarks are those described by a guide who has been there and seen the territory. In the current situation, alas, no such guide is available, and the territory itself may prove to be terra ficta rather than terra firma. A speculative essay of this kind, however cautiously phrased, is bound to contain ideas that will seem childishly naive 10,20 or 50 years hence. One can hope, however, that further attention to the basic biology of the aging process, and the links that connect aging to late-life disease, will hasten the day when we can identify and discard the dead-end ideas and begin to earn our keep in a newly fledged science of applied biogerontology.

\section{ACKNOWLEDGMENTS}

The author thanks Steven Austad for his unpublished calculations on species lifespans in relation to fibroblast growth limits and for valuable tutorials on comparative biology; Kevin Flurkey and David Harrison for unpublished results on the longevity of dw/dw mice; Roger McCarter for data on the athletic prowess of famished rats; and David Burke and Vince Cristofalo for comments on the manuscript.

\section{REFERENCES}

1. Masoro EJ. Aging: current concepts. In: Masoro EJ, ed. Handbook of Physiology. Section 11: Aging. New York: Oxford University Press, 1995.

2. Austad SN, Fischer KE. Mammalian aging, metabolism, and ecology: Evidence from the bats and marsupials. J Gerontol Biol Sci 1991;46:B47-53.

3. Austad SN. Retarded senescence in an insular population of Virginia opossums. J Zool Lond 1993;229:695-708.

4. Finlay BL, Darlington RB. Linked regularities in the development and evolution of mammalian brains. Science 1995;268:1578-1584.

5. Weindruch R, Walford RL. The retardation of aging and disease by dietary restriction. Springfield, IL: Charles C Thomas, 1988.

6. Williams GC. Pleiotropy, natural selection, and the evolution of senescence. Evolution 1957;11:398-411.

7. Hutchinson EW, Rose MR. Quantitative genetics of postponed aging in Drosophila melanogaster. I. Analysis of outbred populations. Genetics $1991 ; 127: 719-727$.

8. Luckinbill LS, Arking R, Clare MJ et al. Selection for delayed senescence in Drosophilia melanogaster. Evolution 1984;38:996-1003.

9. Promislow DE. On size and survival: Progress and pitfalls in the allometry of life span. J Gerontol 1993;48:B115-123.

10. Austad SN. Comparative aging and life histories in mammals. Exp Gerontol 1997;32:23-38.

11. Austad SN, Fischer KE. Primate longevity: Its place in the mammalian scheme. Am J Primatol 1992;28:251-261.

12. $\mathrm{Ku} \mathrm{HH}$, Sohal RS. Comparison of mitochondrial pro-oxidant generation and anti-oxidant defenses between rat and pigeon: Possible basis of variation in longevity and metabolic potential. Mech Ageing Dev 1993;72:67-76.

13. Reznick DN, Shaw FH, Rodd FH et al. Evaluation of the rate of evolution in natural populations of guppies (Poecilia reticulata). Science 1997;275:19341937.

14. Kenyon C, Chang J, Gensch E et al. A C.elegans mutant that lives twice as long as wild type. Nature 1993;366:461-464.

15. Larsen PL, Albert PS, Riddle DL. Genes that regulate both development and longevity in Caenorhabditis elegans. Genetics 1995;139:1567-1583.

16. Orr WC, Sohal RS. Extension of life-span by overexpression of superoxide dismutase and catalase in Drosophila melanogaster. Science 1994;263:1128-1130.

17. Yu CE, Oshima J, Fu YH et al. Positional cloning of the Warner's syndrome gene. Science 1996;272:258-262.

18. Harrison DE. Potential misinterpretations using models of accelerated aging. J Gerontol Biol Sci 1994;49:B245.

19. Partridge L, Harvey PH. Methusalah among nematodes. Nature 1993;366:404-405.

20. Brown-Borg HM, Borg KE, Meliska CJ et al. Dwarf mice and the ageing process. Nature 1996;384:33.

21. Li Y, Deeb B, Pendergrass W et al. Cellular proliferative capacity and life span in small and large dogs. J Gerontol A Biol Sci. Med. Sci. 1996;51:B403-408.

22. Johnson TE, Lithgow GJ, Murakami S. Hypothesis: Interventions that increase the response to stress offer the potential for effective life prolongation and increased health. J Gerontol A Biol Sci Med Sci 1996;51:B392-395.

23. Costa PT, McCrae RR. Design and analysis of aging studies. In: Masoro EJ, ed. Handbook of Physiology. Section 11: Aging. New York: Oxford University Press, 1995.

24. Short R, Williams DD, Bowden DM. Circulating antioxidants as determinants of the rate of biological aging in pigtailed macaques (Macaca nemestrina). J Gerontol Biol Sci 1997;52A:B26-38.

25. Yu BP. Putative Interventions. In: Masoro EJ, ed. Handbook of Physiology. Section 11: Aging. New York: Oxford University Press, 1995, pp 613-631.

26. Weindruch $\mathrm{R}$, Walford RL. Dietary restriction in mice beginning at 1 year of age: Effect on life span and spontaneous cancer incidence. Science 1982;215:1415-1418.

27. Masoro EJ. Food restriction in rodents: An evaluation of its role in the study of aging. J Gerontol 1988;43:B59-64.

28. McCarter $\mathrm{R}$, Masoro EJ, Yu BP. Does food restriction retard aging by reducing the metabolic rate? Am J Physiol 1985;248:E488-490.

29. Merry BJ, Holehan AM. Onset of puberty and duration of fertility in rats fed a restricted diet. J Reprod Fertil 1979;57:253-259.

30. Masoro EJ, McCarter RJ, Katz MS et al. Dietary restriction alters characteristics of glucose fuel use. J Gerontol 1992;47:B202-208. (Published erratum appears in J Gerontol 1993 Mar;48(2):B73).

31. Sabatino F, Masoro EJ, McMahan CA et al. Assessment of the role of the glucocorticoid system in aging processes and in the action of food restriction. J Gerontol Biol Sci 1991;46:B171-179.

32. Heydari AR, Cunrad CC, Richardson A. Expression of heat shock genes in 
hepatocytes is affected by age and food restriction in rats. J Nutr 1995;125:410-418.

33. Yu BP, Lee DW, Marler CG et al. Mechanism of food restriction: Protection of cellular homeostasis. Proc Soc Exp Biol Med 1990;193:13-15.

34. Kemnitz JW, Weindruch R, Roecker EB et al. Dietary restriction of adult male rhesus monkeys: design, methodology, and preliminary findings from the first year of study. J Gerontol 1993;48:B17-26.

35. Lane MA, Ball SS, Ingram DK et al. Diet restriction in thesus monkeys lowers fasting and glucose-stimulated glucoregulatory end points. Am J Physiol 1995;268:E941-948.

36. Bodkin NL, Ortmeyer $\mathrm{HK}$, Hansen BC.: Long-term dietary restriction in older-aged rhesus monkeys: Effects on insulin resistance. J Gerontol A Biol Sci Med Sci 1995;50:B142-147.

37. Olshansky SJ, Carnes BA, Cassel C. In search of Methuselah: Estimating the upper limits to human longevity. Science 1990;250:634-640.

38. Covelli V, Mouton D, Di Majo V et al. Inheritance of immune responsiveness, life span, and disease incidence in interline crosses of mice selected for high or low multispecific antibody production. J Immunol 1989;142:122412.34.

39. Gelman R, Watson A, Bronson $\mathrm{R}$ et al. Murine chromosomal regions correlated with longevity. Genetics 1988;118:693-704.

40. O'Brien SJ, Roelke ME, Marker L et al, Genetic basis for species vulnerability in the cheetah. Science 1985;227:1428-1434.

41. Ebihara S, Marks T, Hudson DJ et al. Genetic control of melatonin synthesis in the pineal gland of the mouse. Science 1986;231:491-493.

42. Hayflick $L$, Moorhead PS. The serial cultivation of human diploid cell strains. Exp Cell Res, 1961;25:585-621.

43. Campisi J, Dimri G, Hara E. Control of replicative senescence. In: Schneider EL, Rowe JW, eds. Handbook of the Biology of Aging. New York: Academic Press, 1996, pp 121-149.

44. Goldstein S. Replicative senescence: The human fibroblast comes of age. Science 1990;249:1129-1133.

45. Pereira-Smith OM, Smith JR. Genetic analysis of indefinite division in human cells: Identification of four complementation groups. Proc Natl Acad Sci USA 1988;85:6042-6046.
46. Vaziri $\mathrm{H}$, Schachter $\mathrm{F}$, Uchida I et al. Loss of telomeric DNA during aging of normal and trisomy 21 human lymphocytes. Am J Hum Genet $1993 ; 52: 661-667$.

47. Dimri GP, Lee $X$, Basile $G$ et al. A biomarker that identifies senescent human cells in culture and in aging skin in vivo. Proc Natl Acad Sci USA 1995;92:936.3-9367.

48. Campisi J. Aging and cancer: The double-edged sword of replicative senescence. J Am Geriatr Soc 1997;45:482-488.

49. Wang E. Senescent human fibroblasts resist programmed cell death, and failure to suppress bcl2 is involved. Cancer Res 1995;55:2284-2292.

50. Harley $\mathrm{CB}$, Futcher $\mathrm{AB}$, Greider $\mathrm{CW}$. Telomeres shorten during ageing of human fibroblasts. Nature 1990;345:458-460.

51. Weng NP, Levine BL, June $\mathrm{CH}$ et al. Human naive and memory T lymphocytes differ in telomeric length and replicative potential. Proc Natl Acad Sci USA 1995;92:11091-11094.

52. Chang $E$, Harley $C B$. Telomere length and replicative aging in human vascular tissues. Proc Natl Acad Sci USA 1995;92:11190-11194.

53. Chiu CP, Harlcy CB. Replicative senescence and cell immortality: The role of telomeres and telomerase. Proc Soc Exp Biol Med 1997;214:99-106.

54. Kim NW, Piatyszek MA, Prowse KR et al. Specific association of human telomerase activity with immortal cells and cancer. Science 1994;266:20112015.

55. Hiyama K, Hirai Y, Kynizumi S et al. Activation of telomerase in human lymphocytes and hematopoietic progenitor cells. J Immunol 1995;155:37113715 .

56. van Steensel B, de Lange T. Control of telomere length by the human telomeric protein TRF1. Nature 1997;385:740-743.

57. Smith JR, Pereira-Smith OM. Replicative senescence: implications for in vivo aging and tumor suppression. Science 1996;273:63-67.

58. Rohme D. Evidence for a relationship between longevity of mammalian species and life-spans of normal fibroblasts in vitro and erythrocytes in vivo. Proc Natl Acad Sci USA 1981;78:5009-5013.

59. McCarter RJM, Shimokawa 1, Ikeno Y et al. Physical activity as a factor in the action of dietary restriction on aging: Effects in Fischer 344 rats. Aging Clin Exp Res 1997;9:73-79. 